\title{
Semantic word priming in the absence of eye fixations: Relative contributions of overt and covert attention
}

\author{
Manuel G. Calvo and M. Dolores Castillo \\ University of La Laguna, Tenerife, Spain
}

\begin{abstract}
In the present study, we investigated the role of covert and overt attention in word identification. In repetition and semantic priming paradigms, prime words were followed by a probe for lexical decision. To make the primes available only to covert attention, we presented them for $150 \mathrm{msec}$, parafoveally $\left(2.2^{\circ}\right.$ away from fixation), and under gaze-contingent foveal masking. To make the primes available to overt attention, we presented them for $150 \mathrm{msec}$, at fixation, with no masking. Results showed both repetition and semantic priming in the absence of eye fixations on the primes: There was facilitation for identical and semantically related probe words, relative to an unrelated prime-probe condition. This revealed that both word form and meaning can be processed by covert attention alone. The pattern of relative contributions of covert $(\sim 25 \%)$ and overt $(\sim 75 \%)$ attention was similar for repetition and semantic priming.
\end{abstract}

There is an important debate in cognitive psychology about whether attention is necessary for word identification. A distinction has been made between central attention as a general-purpose resource for cognitive operations, and spatial attention, which involves selective processing of specific locations or stimuli (Ruthruff, Allen, Lien, \& Grabbe, 2008). Regarding central attention, in single-task studies, there is evidence that semantic activation is automatic, in that it is unaffected by consciousness or the intention to understand the word meaning, and by the amount of attentional resources (Neely \& Kahan, 2001). In dualtask paradigms, whereas some studies have concluded that word processing is automatic (Cleland, Gaskell, Quinlan, \& Tamminen, 2006), others have shown that it requires access to central attentional resources (Lien, Ruthruff, Cornett, Goodin, \& Allen, 2008), although this depends on individual differences, such as in reading ability (Ruthruff et al., 2008).

The present study is concerned with the role of spatial attention in word recognition. In spatial attention paradigms, prime words appear at locations separate (vertically displaced or lateralized) from probes or visually capturing cues. Some studies have found word priming (e.g., Kanne, 2002; Marzouki \& Grainger, 2008; Ortells, Abad, Noguera, \& Lupiáñez, 2001; Tse \& Neely, 2007). However, Lachter, Forster, and Ruthruff (2004) have argued that uncontrolled deployment of attention may have occurred in studies showing processing of spatially "unattended" words. By precuing attention to a location above the prime, presenting the prime for only $55 \mathrm{msec}$, and using masks before and after the prime, these authors used conditions that prevented attention to the prime words.
There was facilitation in lexical-decision times for probe words following identical, relative to unrelated, prime words when the primes appeared for 110 or $165 \mathrm{msec}$, but not when they appeared for $55 \mathrm{msec}$. By assuming that shifts of attention to the primes could be performed in the 110- and 165-msec, but not in the 55-msec, displays, Lachter et al. suggested that the observed priming was due to attentional slippage to the primes. They concluded that spatial attention is required, that there is no word identification without attention. In the present study, we attempted to extend two key issues raised by this conclusion: the role of each of two spatial attention mechanisms, and the nature of word identification.

Regarding the first issue, a distinction can be made between overt and covert spatial attention. Overt attention relies on eye fixations on the stimuli, whereas covert attention involves neural adjustments without eye movements (Wu \& Remington, 2003). There is a natural relationship between these mechanisms, with covert attention assisting in the preprocessing of information in the visual periphery where the eyes are to be directed (Findlay \& Gilchrist, 2003). Thus, covert shifts are thought to be faster $(50-100 \mathrm{msec})$ than overt saccades $(150 \mathrm{msec}$; see Lachter et al., 2004; Rayner, 1998). We aim to examine the implications of this distinction. Lachter et al. used a procedure that prevented both covert and overt attention in the 55 -msec prime display. The findings from the absence of priming in this condition indeed suggested that some form of attention is required for word identification, but raised the issue of which attentional mechanism is necessary, or whether both are needed. With the 110- and $165-\mathrm{msec}$ displays, priming effects emerged. It is, however, unclear

M. G. Calvo, mgcalvo@ull.es 
whether they were due to covert attentional shifts only or involved eye fixations as well.

Regarding the second issue, Lachter et al. (2004) chose a repetition-priming paradigm (same prime-probe word) intentionally, in order to maximize sensitivity to attentional leakage, given that repetition priming is typically stronger than semantic priming. Nevertheless, although repetition priming is sensitive to word meaning, it can be affected by word form (orthographic and phonological codes). Accordingly, it would be useful to determine the extent to which covert and overt attention affect the semantic component specifically. The distinction between repetition and semantic priming is further relevant, because the former is less affected by attentional manipulations than the latter (Fabre, Lemaire, \& Grainger, 2007). Furthermore, given that orthographic codes are more likely to be extracted from parafoveal words than are semantic codeswhich may, actually, not be obtained (Altarriba, Kambe, Pollatsek, \& Rayner, 2001; Rayner, 1998) - it is expected that the contribution of covert attention will be greater in repetition than in semantic priming.

In order to address these two issues, we first investigated whether word identification could be accomplished by means of covert attention alone, in the absence of overt attention. To this end, our experimental conditions selectively allowed only covert attention, by preventing eye fixations on the prime, or both overt and covert attention. In the covert-attention-only (henceforth, covert-attention) condition, prime words were presented parafoveally $\left(2.2^{\circ}\right.$ away from fixation), briefly $(150 \mathrm{msec})$, and under foveal gaze-contingent masking, so that the prime was masked by Xs when the viewer initiated saccades to it. Accordingly, the prime was available to covert attention, but could not be fixated, thus preventing overt attention to it. In these conditions, we assumed that the prime word would be initially unattended, but might then capture attention. In the covertplus-overt-attention (henceforth, overt-attention) condition, prime words were presented at fixation (foveally), for $150 \mathrm{msec}$ with no mask. Second, we investigated whether the contribution of covert attention would encompass not only word form, but also word meaning. To this end, we combined repetition- and semantic-priming paradigms. In a lexical-decision task, participants responded whether a probe letter string was a word or not. A condition in which the probe was preceded by an unrelated prime word was compared with one in which the probe was preceded by an identical (repetition priming) or a semantically associated (semantic priming) word. Priming effects in the masked (vs. nonmasked) and the associated (vs. identical) conditions provided information about the specific contribution of covert attention to semantic processing.

\section{METHOD}

\section{Participants}

Forty-eight undergraduates (36 females) participated for course credit.

\section{Stimuli}

Seventy-eight target words were used as probes (word frequency, $M=90.60$; Davis \& Perea, 2005; word length, $M=5.14$ letters).
Another 78 words that were lexical associates of the probe words were used as primes for the prime-probe associated condition (e.g., wood-tree), and an additional 78 words were used for the unrelated condition (e.g., wine-tree). The unrelated words were matched in length $(M=5.17)$ and frequency $(M=68.95)$ with the associated words (length, $M=5.19$; frequency, $M=71.87$ ).

The target words were selected from a larger sample of three groups of 134 words each. The actual priming effects for each pair of associated-probe words were assessed in a lexical-decision task, with the primes and the probes appearing at fixation. Forty-two new participants were presented each of the 134 probe words, with one third preceded by either the same word, the lexical associate, or an unrelated word as primes, for $150 \mathrm{msec}$. Seventy-eight prime-probe pairs were selected when a priming effect of $15 \mathrm{msec}$ or more was obtained in the associated, relative to the unrelated, condition.

\section{Apparatus and Procedure}

Stimuli were presented on a 21 -in. monitor with a $120-\mathrm{Hz}$ refresh rate. Eye movements were recorded with an EyeLink II tracking device (SR Research, Mississauga, ON). This is an infrared system that samples pupil location at a $500-\mathrm{Hz}$ rate, with a spatial accuracy better than $0.5^{\circ}$

The prime words (lowercase) subtended a visual angle between $1.3^{\circ}$ and $1.8^{\circ}$ horizontally; the probe string (uppercase) subtended between $1.4^{\circ}$ and $2.0^{\circ}$. The viewing distance was $60 \mathrm{~cm}$. The participants responded to the probe by pressing one of two keys for "word" and for "nonword." On each trial (see Figure 1), a central circle $\left(0.8^{\circ}\right)$ was initially presented. When the viewer fixated this circle, the prime display appeared for $150 \mathrm{msec}$, with one parafoveal word to the left or right (displaced $\left.2.2^{\circ}\right)$ of a central string of $\mathrm{xx}+\mathrm{xx}\left(1.4^{\circ}\right)$ replacing the circle. After a 150-msec interval, a probe appeared at fixation. Participants were instructed to look at the center. A gaze-contingent display change was implemented: The initial and the final $\mathrm{X}$ of the string of $\mathrm{xx}+\mathrm{xx}$ constituted boundaries; when the foveal fixation crossed either of these boundaries, the parafoveal prime word turned to a mask of Xs. The probe-word:nonword ratio was $0.67: 0.33$.

\section{Design}

Prime-probe relatedness (identical vs. associated vs. unrelated) was a within-subjects factor, and attention condition (covert vs. overt, each with 24 participants, randomly assigned) was a betweensubjects factor. In the identical condition, the same word was presented as a prime and a probe. In the associated condition, the prime and the probe were related in meaning, but not in orthography or phonology. In the unrelated condition, the prime and the probe were different in meaning and form. In the covert-attention condition, the prime word was presented parafoveally and under gaze-contingent foveal masking. In the overt-attention condition, the prime appeared at fixation and was unmasked. Of all trials performed by each participant, one third involved identical primes, one third involved associated primes, and one third involved unrelated primes. Each probe was preceded by a different prime. In the covert-attention condition, half of the primes were presented in the left visual field and the other half were presented in the right visual field.

\section{Measures}

Response accuracy and reaction times for correct responses in the lexical-decision task were measured. In the covert-attention condition, the probability of saccades to the prime, saccade latencies (i.e., the time to initiate an eye movement from the central fixation point toward the prime), and saccade end time (i.e., the time taken to land on the location of the prime) were also assessed.

\section{RESULTS}

\section{Lexical-Decision Performance}

Response accuracy and reaction times were analyzed using a 3 (prime-probe relatedness) $\times 2$ (prime loca- 


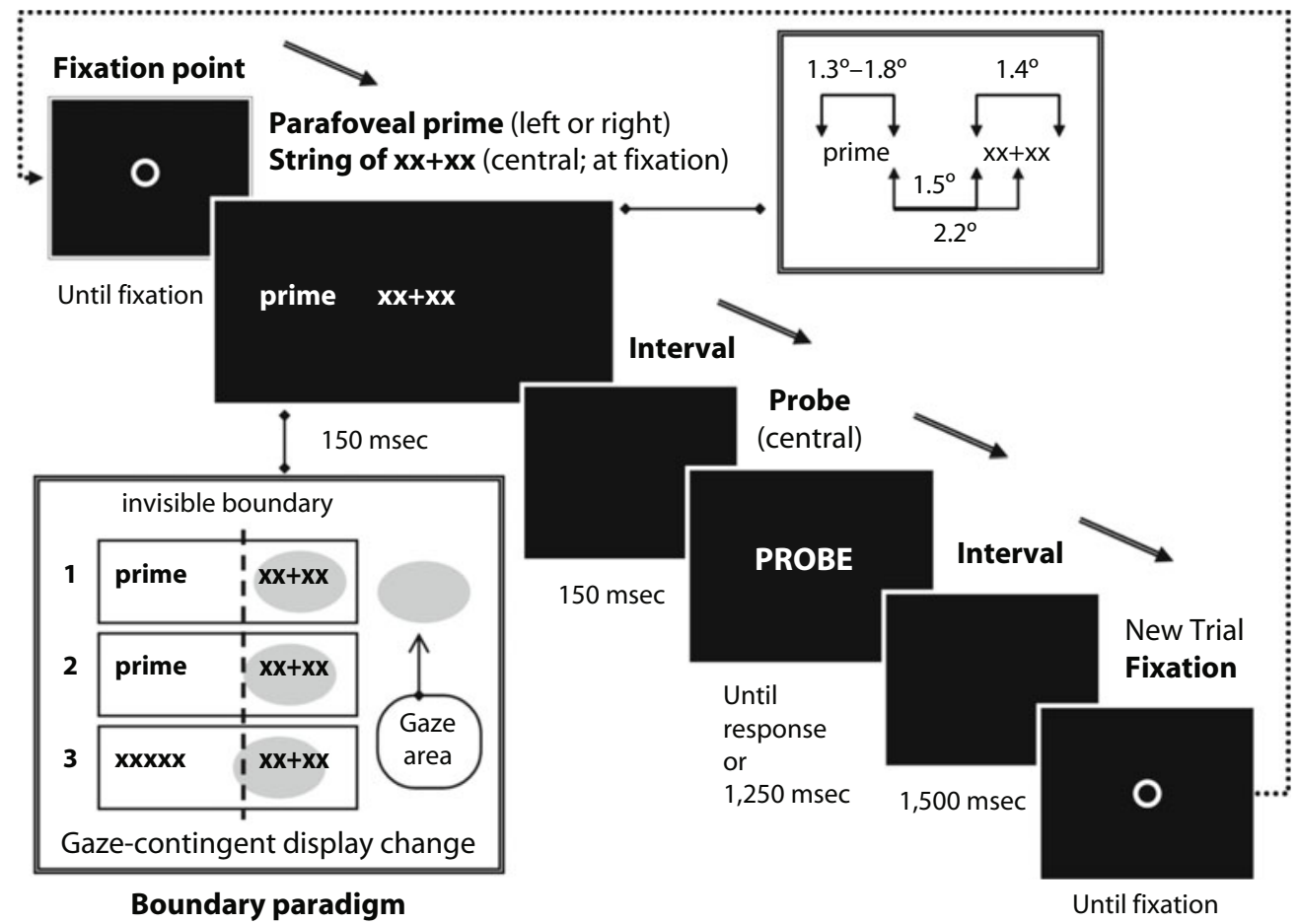

Figure 1. Sequence of events on each trial in the covert attention condition (parafoveal, foveally masked, prime). In the overt attention condition, the prime appeared centrally at fixation, with no gaze-contingent masking.

tion) ANOVA. For accuracy, there were main effects of relatedness $[F(2,92)=5.81, p<.01]$, but not of location $(F<1)$, and an interaction $[F(2,92)=4.41, p<.025]$. For reaction times, the effects of relatedness $[F(2,92)=$ $141.77, p<.0001]$ and location $[F(1,46)=8.11, p<$ $.01]$ were qualified by an interaction $[F(2,92)=57.00$, $p<.0001]$. To decompose the interactions, we conducted one-way ANOVAs separately for each attention condition, with relatedness as a factor and Bonferroni corrections for multiple contrasts (see mean scores in Figure 2).

When the primes were presented parafoveally and masked (i.e., covert-attention condition), response accuracy was equivalent in the identical $(M=.981)$, associated $(M=.979)$, and unrelated $(M=.977)$ conditions. The analysis of reaction times yielded main effects of relatedness $[F(2,46)=12.16, p<.0001]$, with faster responses in the identical $(M=631 \mathrm{msec}, p<.001)$ and associated $(M=642 \mathrm{msec}, p<.05)$ conditions than in the unrelated $(M=661 \mathrm{msec})$ condition. The difference between the identical and the associated condition was nonsignificant $(p=.17)$.

When the primes were presented at fixation and unmasked (i.e., overt-attention condition), effects on accuracy $[F(2,46)=9.94, p<.0001]$ revealed differences between the identical $(M=.994)$ and unrelated $(M=.953$, $p<.01)$ conditions and between the associated $(M=$ $.992)$ and unrelated $(p<.025)$ conditions. For response latencies $[F(2,46)=156.87, p<.0001]$, there were differences among all three conditions (all $p \mathrm{~s}<.0001$ ). Responses were $66 \mathrm{msec}$ faster in the identical than in the associated condition, in which responses were $68 \mathrm{msec}$ faster than those in the unrelated condition.

\section{Eye Movement Data}

In the covert-attention condition, eye movement variables were analyzed using a 3 (relatedness) $\times 2$ (visual field) ANOVA. The probability of initiated saccades toward the prime word $(M=.145$, or $14.5 \%$ of the trials; $S D=0.19)$, the mean saccade latency $(134.5 \mathrm{msec}, S D=$ $9.17)$, and the mean saccade end time $(160 \mathrm{msec}, S D=$ 19.53) did not vary as a function of relatedness or of visual field (all $F_{\mathrm{S}}<1$ ). Of those trials in which there were initiated saccades, the percentage of saccade end times shorter than $150 \mathrm{msec}$ was $24 \%$. This means that in less than $2 \%$ of the total number of trials, including those with no saccades, were there fixations on the prime location. For these few cases, the mean duration of fixations on the prime area was $12 \mathrm{msec}$. Nevertheless, when this occurred, the gaze-contingent display change masked the prime word, which could not be foveally fixated.

In sum, in the condition that allowed only covert attention, lexical-decision responses were faster when the probe was associated (and also when it was identical) than when it was unrelated to the prime word. This reveals that there was true semantic priming (and not merely repetition priming), and implies that the meaning of the prime word was processed.

Priming occurred in the absence of eye fixations on the prime words. There was no overt attention, given the extremely low number of otherwise very short fixations on the location of the prime word, which, when this happened, 


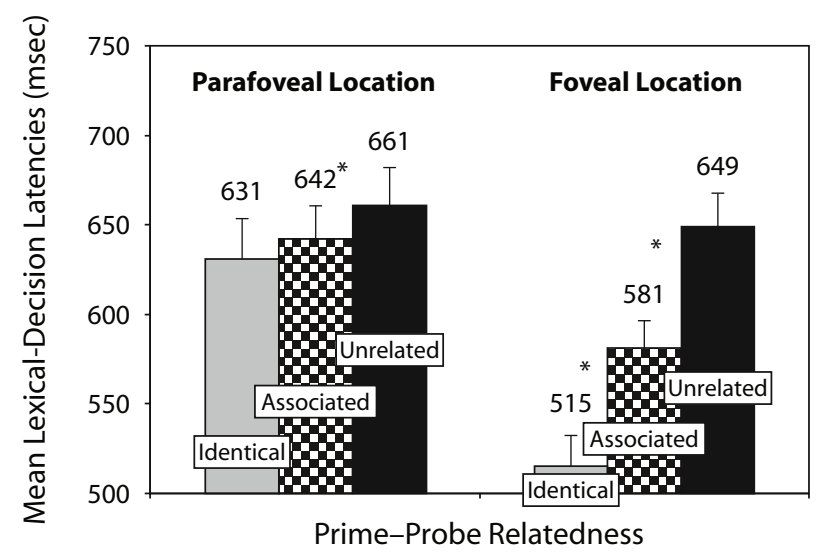

Figure 2. Mean correct response times (in milliseconds) to probes following an identical, a lexical associate, or an unrelated prime word, in the parafoveal-masked (averaged across visual fields) or the foveal-unmasked locations. Asterisks show significant differences.

was foveally masked. Accordingly, the primes must have been processed by covert attention, which, therefore, would have made a specific contribution to word identification.

\section{Relative Contributions of Covert and Overt Attention to Semantic and Repetition Priming}

We computed priming scores (RTs unrelated - RTs identical $=$ repetition priming; RTs unrelated - RTs associated = semantic priming; see Figure 3). Assuming that both overt and covert attention to the prime are available under the foveal-unmasked presentation, whereas only covert attention is possible under the parafoveal-masked presentation, the priming scores provide an estimate of the relative role of each attentional mechanism to word identification. Repetition priming was greater $[t(46)=10.97$, $p<.0001]$ in the foveal-unmasked than in the parafovealmasked condition (a 134- vs. 30-msec facilitation effect, respectively), which were both statistically significant, as indicated in the separate analyses for each condition, above. Similarly, semantic priming was greater $[t(46)=4.60, p<$ $.0001]$ in the foveal-unmasked than in the parafovealmasked condition (68 vs. $19 \mathrm{msec}$, respectively; both were significant, as indicated in the separate analyses).

If we take the 134-msec (repetition priming) and the 68-msec (semantic priming) scores in the foveal-unmasked condition as the total (100\%) joint effect of overt and covert attention, this implies that the specific contribution of covert attention was $22 \%$ (30 $\mathrm{msec})$ to repetition priming and $28 \%(19 \mathrm{msec})$ to semantic priming. The difference $(134-30=104 \mathrm{msec}$, and $68-19=49 \mathrm{msec}$, respectively) could be attributed to overt attention. Of course, these are only rough estimates, but they reveal a significant, unique involvement of covert attention, although that of overt attention is (not surprisingly) greater. ${ }^{1}$

\section{DISCUSSION}

We examined the role of covert attention, relative to overt attention, in word identification. The major results showed that overtly unattended words (due to foveal masking) produced both repetition and semantic priming if they could be covertly attended (in parafoveal vision): There was facilitation in lexical decision on both an identical probe and a semantically related probe, relative to an unrelated prime-probe condition. This indicates that not only word form (e.g., orthography) but also meaning can be processed by covert attention alone. In addition, although priming was stronger when the prime words were available to overt attention, the contribution of covert attention was similar for repetition and semantic priming. These findings add to those from prior research on the role of attention in word identification.

Regarding attentional involvement, these results do not dispute Lachter et al.'s (2004) claim that attention is necessary for word identification. Rather, they refine this conclusion by showing that overt attention is not necessary if covert attention is available. Some form of attention may be required, but not both. In prior research, spatial attention had been prevented by presenting the unattended words briefly and away from the current fixation, assuming that there was no time for saccades. This was achieved by means of presenting a prime and a probe simultaneously, but spatially separated (Tse \& Neely, 2007), or by the use of precuing paradigms (Ortells et al., 2001). In these conditions, prime words at irrelevant locations have been found to facilitate the processing of a related probe. It is likely that the priming effects were due to covert attention, a finding that is consistent with the present findings. However, the extent to which the procedures used to prevent attention affected the overt and/or covert mechanisms was never determined in previous studies. The assumption that eye movements to prime words did not occur - although probably correct - was not checked. As a consequence, the roles of overt and covert attention could not be separated. By preventing eye fixations by using foveal-contingent masking of otherwise parafoveally accessible prime words, our data have isolated the

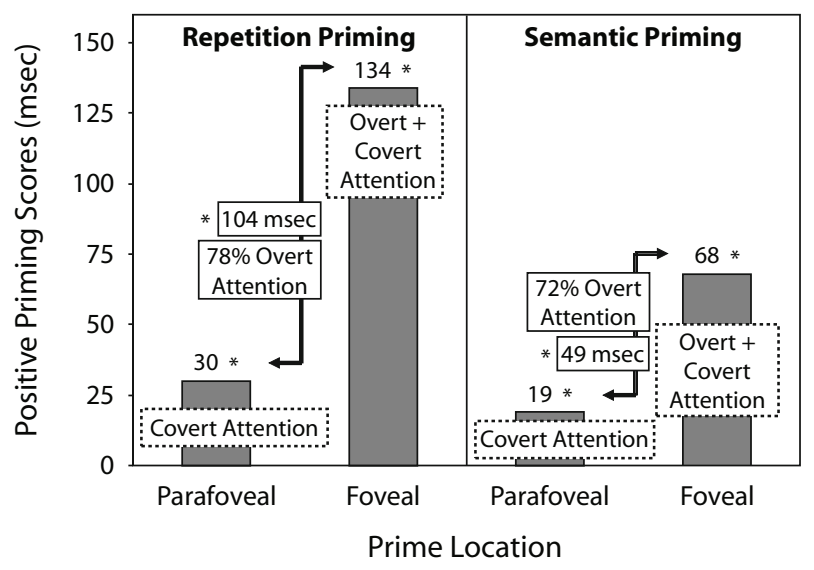

Figure 3. Repetition priming scores (i.e., unrelated - identical lexical-decision times), and semantic priming scores (i.e., unrelated - associated lexical-decision times), as a function of attention. Positive scores indicate facilitation in the identical primeprobe condition or in the associated prime-probe condition. Asterisks show significant differences. 
specific contribution of covert attention. A recent study by Marzouki and Grainger (2008) has confirmed that repetition priming effects can occur in the absence of fixations on eccentric prime words. Our study adds to that finding by extending the effects to semantic priming.

Regarding processing of word meaning, our findings are consistent with those obtained in studies using semantic-priming paradigms in which eye fixations were not assessed or prevented (e.g., Ortells et al., 2001; Tse $\&$ Neely, 2007). The demonstration of semantic priming from words that were not fixated is one of the main contributions of the present study. Prior studies using precuing or forward- and backward-masking paradigms, but not assessing actual eye movements and not implementing gaze-contingent masking during the prime display, could not rule out the involvement of overt attention. Our results indicate that the meaning of words can be processed when available only to covert attention. This finding is, however, not consistent with data from an eye movement study in which gaze-contingent masking was also used, and orthographic - but not semantic - priming was found (Altarriba et al., 2001). The inconsistency can, nonetheless, be explained as a function of reduced semantic sensitivity in the Altarriba et al. paradigm. The reason is that parafoveal Spanish words served as primes for Englishtranslation probe words in bilingual participants. In fact, Altarriba et al. considered the possibility that translation processes involve code switching, which may weaken semantic priming. In our study, the empirical criterion used for stimulus preselection ensured that the associated words did produce reliable priming. Accordingly, semantic priming can occur through covert attention, at least for word pairs that also show reliable priming when they are available to overt attention.

The present findings have theoretical implications for the distinction of covert and overt attention, as well as for their role in orthographic and semantic word processing. First, there is consensus that covert attention involves attention to extrafoveal stimuli in the absence of eye movements, whereas overt attention relies on fixations on the stimuli (Findlay \& Gilchrist, 2003; Wu \& Remington, 2003). An important issue is whether these two forms of attention are qualitatively different or, rather, involve merely quantitative differences, where overt attention includes covert attention plus the sensory and perceptual benefits of foveation. The present findings support the latter view, based on the result that the foveal:parafoveal ratio was equivalent for repetition and semantic priming. The greater absolute priming effects in the overt condition could thus be due to more attention than in the covert condition. If the two forms of attention were qualitatively different, we should have found their relative contributions to repetition and semantic priming to be different as well. This conceptualization is consistent with neurophysiological evidence that indicates that both forms of attention are controlled by a common set of mechanisms, with overlapping brain areas (Ignashchenkova, Dicke, Haarmeier, \& Thier, 2004).

Second, we initially predicted a larger effect of covert attention on repetition than on semantic priming (see the introduction), but the actual findings have revealed a similar influence. Prior research has shown that word-form codes are more likely than semantic codes to be obtained parafoveally (Altarriba et al., 2001; Rayner, 1998). The present findings indicate that the relative contribution of covert attention to repetition and semantic priming is comparable; overt attention was not more necessary for semantic than for repetition priming. Although in absolute terms semantic priming was lower than repetition priming, semantic priming was not lower in the covert, relative to the overt, attention condition than was repetition priming. This suggests that, once word form is identified (or the orthographic codes are extracted) parafoveally, semantic processing starts as quickly as when these are obtained foveally. This is consistent with neurophysiological evidence that both repetition and semantic priming are modulated by at least partially overlapping neural mechanisms (Pesciarelli et al., 2007).

In sum, identification of both word form and meaning can be accomplished in the absence of fixations on (or overt attention to) the word. The relative contribution of covert attention is similar for identical and semantic priming, although overt attention makes a stronger contribution, in absolute terms. Some kind of attention is probably required for word identification, but overt attention is not indispensable.

\section{AUTHOR NOTE}

This research was supported by Grant SEJ2004-420/PSIC from the Spanish Ministry of Education and Science. Correspondence concerning this article should be sent to M. G. Calvo, Department of Cognitive Psychology, University of La Laguna, 38205, Tenerife, Spain (e-mail: mgcalvo@ull.es).

\section{REFERENCES}

Altarriba, J., Kambe, G., Pollatsek, A., \& Rayner, K. (2001). Semantic codes are not used in integrating information across eye fixations in reading: Evidence from fluent Spanish-English bilinguals. Perception \& Psychophysics, 63, 875-890.

Cleland, A. A., Gaskell, M. G., Quinlan, P. T., \& Tamminen, J. (2006). Frequency effects in spoken and visual word recognition: Evidence from dual-task methodologies. Journal of Experimental Psychology: Human Perception \& Performance, 32, 104-119.

Davis, C. J., \& Perea, M. (2005). BuscaPalabras: A program for deriving orthographic and phonological neighborhood statistics and other psycholinguistic indices in Spanish. Behavior Research Methods, 37, 665-671.

Fabre, L., Lemaire, P., \& Grainger, J. (2007). Attentional modulation of masked repetition and categorical priming in young and older adults. Cognition, 105, 513-532.

Findlay, J. M., \& Gilchrist, I. D. (2003). Active vision: The psychology of looking and seeing. Oxford: Oxford University Press.

Ignashchenkova, A., Dicke, P. W., HaArmeier, T., \& Thier, P. (2004). Neuron-specific contribution of the superior colliculus to overt and covert shifts of attention. Nature Neuroscience, 7, 56-64.

KANNE, S. M. (2002). The role of semantic, orthographic, and phonological prime information in unilateral visual neglect. Cognitive Neuropsychology, 19, 245-261.

Lachter, J., Forster, K. I., \& Ruthruff, E. (2004). Forty-five years after Broadbent (1958): Still no identification without attention. Psychological Review, 111, 880-913.

Lien, M.-C., Ruthruff, E., Cornett, L., Goodin, Z., \& Allen, P. A. (2008). On the nonautomaticity of visual word processing: Electrophysiological evidence that word processing requires central attention. Journal of Experimental Psychology: Human Perception \& Performance, 34, 751-773. 
Marzouki, Y., \& Grainger, J. (2008). Effects of prime and target eccentricity on masked repetition priming. Psychonomic Bulletin \& Review, 15, 141-148.

NeEly, J. H., \& KaHAN, T. A. (2001). Is semantic activation automatic? A critical re-evaluation. In H. L. Roediger III, J. S. Nairne, I. Neath, \& A. M. Surprenant (Eds.), The nature of remembering: Essays in honor of Robert G. Crowder (pp. 69-93). Washington, DC: American Psychological Association.

Ortells, J. J., Abad, M. J. F., Noguera, C., \& Lupiáñez, J. (2001). Influence of prime-probe stimulus onset asynchrony and prime precuing manipulations on semantic priming effects with words in a lexical-decision task. Journal of Experimental Psychology: Human Perception \& Performance, 27, 75-91.

Pesciarelli, F., Kutas, M., Dell'Aqua, R., Peressotti, F., Job, R., \& Urbach, T. P. (2007). Semantic and repetition priming within the attentional blink: An event-related brain potential (ERP) investigation study. Biological Psychology, 76, 21-30.

RAYNER, K. (1998). Eye movements in reading and information processing: 20 years of research. Psychological Bulletin, 124, 372-422.

Ruthruff, E., Allen, P. A., Lien, M.-C., \& Grabbe, J. (2008). Visual word recognition without central attention: Evidence for greater automaticity with greater reading ability. Psychonomic Bulletin \& Review, 15, 337-343.
TsE, C.-S., \& NeELy, J. H. (2007). Semantic priming from letter-searched primes occurs for low- but not high-frequency targets: Automatic semantic access may not be a myth. Journal of Experimental Psychology: Learning, Memory, \& Cognition, 33, 1143-1161.

Wu, S.-C., \& Remington, R. W. (2003). Characteristics of covert and overt visual orienting: Evidence from attentional and oculomotor capture. Journal of Experimental Psychology: Human Perception \& Performance, 29, 1050-1067.

\section{NOTE}

1. The foveal condition probably benefits from attention for a longer period of time than the parafoveal condition, because the latter requires waiting for a shift of attention that is not necessary in the former. Accordingly, the $22 \%-28 \%$ figure for the relative contribution of covert attention probably provides a lower boundary on its contribution. If so, the real contribution of covert attention might actually be greater. This further argues that there is a significant role of covert attention in word identification.

(Manuscript received February 27, 2008; revision accepted for publication August 8,2008 .) 